\title{
New methods to measure residues coevolution in proteins
}

Hongyun Gao ${ }^{1,2}$, Yongchao Dou ${ }^{1,2}$, Jialiang Yang ${ }^{3}$ and Jun Wang ${ }^{4,5^{*}}$

\begin{abstract}
Background: The covariation of two sites in a protein is often used as the degree of their coevolution. To quantify the covariation many methods have been developed and most of them are based on residues position-specific frequencies by using the mutual information (MI) model.

Results: In the paper, we proposed several new measures to incorporate new biological constraints in quantifying the covariation. The first measure is the mutual information with the amino acid background distribution (MIB), which incorporates the amino acid background distribution into the marginal distribution of the $\mathrm{Ml}$ model. The modification is made to remove the effect of amino acid evolutionary pressure in measuring covariation. The second measure is the mutual information of residues physicochemical properties (MIP), which is used to measure the covariation of physicochemical properties of two sites. The third measure called MIBP is proposed by applying residues physicochemical properties into the MIB model. Moreover, scores of our new measures are applied to a robust indicator conn $(k)$ in finding the covariation signal of each site.

Conclusions: We find that incorporating amino acid background distribution is effective in removing the effect of evolutionary pressure of amino acids. Thus the MIB measure describes more biological background information for the coevolution of residues. Besides, our analysis also reveals that the covariation of physicochemical properties is a new aspect of coevolution information.
\end{abstract}

\section{Background}

A protein's function depends on its three-dimensional (3D) structure and interactions of residues [1]. When there is a mutation of functionally or structurally important residues, compensatory mutations may occur to preserve or restore the function or structure of the protein. Thus the knowledge of residues coevolution helps to predict protein function and guide experimental analysis. To quantify the coevolution of a protein chain, a multiple sequence alignment (MSA) of the chain and its homologous sequences are generated at first. Then the covariation of two sites is used as the degree of their coevolution.

In order to quantify the covariation of two sites in a given MSA, many computational methods have been developed in recent years. These methods can be divided into two groups: parametric methods and

\footnotetext{
* Correspondence: jwang@shnu.edu.cn

${ }^{4}$ Scientific Computing Key Laboratory of Shanghai Universities, 200234

Shanghai, People's Republic of China

Full list of author information is available at the end of the article
}

nonparametric ones [2-14]. The parametric methods incorporate maximum likelihood approximations [15], Bayesian probabilities [16], Phylogentic approaches [17] and so on. While nonparametric methods are more extensive and most of them are based on mutual information (MI). The MI model is taken from information theory [18], and uses the position specific distribution of two sites [19-21]. For example, [6] tested the effect of the size of MSAs and the mutation rate on two sources of background (finite sample size effects and phylogenetic influence). They also tested the performance of various normalizations of $\mathrm{MI}$ in enhancing the detection of coevolving sites. To integrate different biological constraints with the MI model, some methods focused on how to relate observation counts to expected distribution [22-24]. However, [25] pointed out that none of the estimates are more 'correct' than others since all the methods merely depend on assumptions. And it has been shown that the estimates of MI are more affected by these assumptions than by the actually observed data. The 'correct' conditional assumptions refer to the

Ciomed Central

(c) 2011 Gao et al; licensee BioMed Central Ltd. This is an Open Access article distributed under the terms of the Creative Commons Attribution License (http://creativecommons.org/licenses/by/2.0), which permits unrestricted use, distribution, and reproduction in any medium, provided the original work is properly cited. 
assumptions strictly matching known biological constraints. Another widely used nonparametric method is ELSC which applies a perturbation-based method to calculate explicitly the likelihood of evolutionary covariance in MSAs [26]. Although many biological constraints have been used in measuring covariation, the amino acid background distribution and their physicochemical properties are ignored in previous methods.

In order to improve covariation measures, two new biological constraints are introduced in the paper. The first constraint is the amino acid background distribution which indicates the evolutionary pressures of amino acids. It has been proved that if a residue is under relatively low evolutionary pressure, it will be readily replaced in the evolutionary history. Thus this residue will have relatively low frequency in an MSA and vice versa. In general, the frequency of a residue can be used as an estimate of its evolutionary pressure and is often called the background frequency of the residue [27]. A residue background distribution is called 'no pressure' if it can describe the distribution of amino acids subject to no evolutionary pressure [28]. Actually, it depicts evolutionary pressures for each residue exactly. However, it is difficult to estimate the 'no pressure' background distribution. Thus statistical background usually provides an alternative to approximate it. As suggested by several existing conservation measures, the BLOSUM62 background distribution [29] is used as the amino acid background distribution in the paper. By incorporating amino acid background distribution into account, we proposed a new method MI with amino acid background distribution (MIB).

The second constraint to improve covariation measures is the physicochemical properties which are important in predicting functional important residues [30-36]. In order to incorporate the physicochemical properties into the MI model, amino acids are often grouped into six disjoint groups or ten overlapping groups in previous works [37]. In the study, the classification of ten overlapping groups of amino acids is chosen since six disjoint groups has a deficiency that residues of different types are treated equally different. Then, a new method called the MI with physicochemical properties (MIP) is developed to estimate the variation of physicochemical properties of two given sites.

The third model is called MIBP method which is used to estimate the covariation of physicochemical properties by removing their background distributions. Moreover, our measures are applied to a robust indicator conn(k) [23] in finding covariation signal of each site.

\section{Methods}

Since the reliability of MI values depends on that of MSA, the quality of MSA is important in measuring the covariation. In the paper, MSAs are downloaded from the PFAM [38] data base. And the number of sequences in an alignment should be greater than 125 [6]. Given an MSA, sequences in it are clustered at $90 \%$ sequence identity and the redundant sequences are removed. Moreover, the columns with more than 25\% gaps are also removed as suggested by [23]. Gaps are also ignored when position specific frequencies are calculated. Moreover, these proposed methods are also tested on a recently published data set which was created by Capra and Singh (2007) [28]. MSAs in the data set with lower than 125 sequences are removed. After filtering, 496 MSAs remained and results on them can be found in the supplement material. Throughout the paper, we use $N$ to denote the number of sequences in the MSA and $c \_$pair $(K, L)$ to denote the pair of column $K$ and column $L$ used to calculate the MI based measures.

\section{The MI model with the amino acid background distribution (MIB)}

An ordering is first specified to the 20 amino acids. Given an MSA, the amino acid position specific frequency of a column $K$ for the $i$ th residue is calculated as:

$$
p\left(K^{i}\right)=\frac{\operatorname{count}\left(K^{i}\right)}{N},
$$

where $\operatorname{count}\left(K^{i}\right)$ denotes the number of the $i$ th $(i=1$, ..., 20) residue in column $K$.

Similarly, the joint probability distribution of $c_{-}$pair $(K, L)$ for a residue pair consisting of the $i$ th and $j$ th residue is defined as:

$$
p\left(K^{i}, L^{j}\right)=\frac{\operatorname{count}\left(K^{i}, L^{j}\right)}{N},
$$

where $\operatorname{count}\left(K^{i}, L^{j}\right)$ denotes the number of rows, in which the residues in column $K$ and $L$ are the $i$ th and jth residue, respectively.

Based on the above definitions, the classical MI [18] is calculated as:

$$
M I(K, L)=\sum_{i=1}^{20} \sum_{j=1}^{20} p\left(K^{i}, L^{j}\right) \log \frac{p\left(K^{i}, L^{j}\right)}{p\left(K^{i}\right) \cdot p\left(L^{j}\right)} .
$$

Clearly, if $p\left(K^{i}, L^{j}\right)=0$, then the value of the monomial $p\left(K^{i}, L^{j}\right) \log \frac{p\left(K^{i}, L^{j}\right)}{p\left(K^{i}\right) \cdot p\left(L^{j}\right)}$ is 0 . Moreover, if one column in a column-pair is fully conserved, then $M I(K$, $L)=0$. As suggested by [6], we further normalize $M I$ $(K, L)$ by $H(K, L)$, and define

$$
M I^{\prime}(K, L)=\frac{M I(K, L)}{H(K, L)}
$$


where $H(K, L)=-\sum_{i=1}^{20} \sum_{j=1}^{20} p\left(K^{i}, L^{j}\right) \log p\left(K^{i}, L^{j}\right)$.

In order to account for the background distribution of amino acids, we introduce the marginal distribution, which is defined as:

$$
p_{b}\left(K^{i}\right)=\frac{\frac{p\left(K^{i}\right)}{q^{i}}}{\sum_{m=1}^{20} \frac{p\left(K^{m}\right)}{q^{m}}},
$$

where $q$ is the BLOSUM62 amino acid background distribution. Then a new estimation of covariation called the MI with the amino acid background distribution (MIB) is defined as:

$$
\operatorname{MIB}(K, L)=\sum_{i=1}^{20} \sum_{j=1}^{20} p\left(K^{i}, L^{j}\right) \log \frac{p\left(K^{i}, L^{j}\right)}{p_{b}\left(K^{i}\right) \cdot p_{b}\left(L^{j}\right)} .
$$

Similarly, if $p\left(K^{i}, L^{j}\right)=0$, then the value of the monomial $p\left(K^{i}, L^{j}\right) \log \frac{p\left(K^{i}, L^{j}\right)}{p_{b}\left(K^{i}\right) \cdot p_{b}\left(L^{j}\right)}$ is considered as 0 .

In addition, we also normalize $M I B(K, L)$ by $H(K, L)$ and define a new measure MIB',

$$
\operatorname{MIB}^{\prime}(K, L)=\frac{\operatorname{MIB}(K, L)}{H(K, L)} .
$$

MIB' is sometimes referred to as the normalized MIB.

\section{The covariation of physicochemical properties}

To measure the covariation of amino acid physicochemical properties, amino acids are grouped into ten overlapping groups as suggested by Taylor [37]. The ten overlapping groups are: hydrophobic $(\mathrm{A}, \mathrm{G}, \mathrm{C}, \mathrm{T}, \mathrm{I}, \mathrm{V}$, L, K, H, F, Y, W, M), aromatic (F, Y, W, H), aliphatic (I, $\mathrm{V}, \mathrm{L}$ ), tiny $(\mathrm{A}, \mathrm{S}, \mathrm{G}, \mathrm{C})$, small $(\mathrm{P}, \mathrm{N}, \mathrm{D}, \mathrm{T}, \mathrm{C}, \mathrm{A}, \mathrm{G}, \mathrm{S}, \mathrm{V})$, proline $(P)$, charged $(K, H, R, D, E)$, negative $(D, E)$, polar $(\mathrm{N}, \mathrm{Q}, \mathrm{S}, \mathrm{D}, \mathrm{E}, \mathrm{C}, \mathrm{T}, \mathrm{K}, \mathrm{R}, \mathrm{H}, \mathrm{Y}, \mathrm{W})$ and positive $(\mathrm{K}, \mathrm{H}, \mathrm{R})$. In this section, we use g_pair $(a, b)$ to denote the group pair, in which the former residue belongs to group $a$ and the latter residue belongs to group $b$.

Then, the fractional frequency of group $a$ in column $K$ is defined as:

$$
p_{p}\left(K^{a}\right)=\frac{\operatorname{count}\left(K^{a}\right)}{N},
$$

where $\operatorname{count}\left(K^{a}\right)$ is the number of residues belongs to group $a$ in column $K$.

The joint property distribution of $c_{-}$pair $(K, L)$ for g_pair $(a, b)$ is defined as:

$$
p_{p}\left(K^{a}, L^{b}\right)=\frac{\operatorname{count}\left(K^{a}, L^{b}\right)}{N},
$$

where the $\operatorname{count}\left(K^{a}, L^{b}\right)$ is the number of residue-pairs belonging to g_pair $(a, b)$.

We define a new measure of covariation as:

$$
\operatorname{MIP}(K, L)=\sum_{a=1}^{10} \sum_{b=1}^{10} p_{p}\left(K^{a}, L^{b}\right) \log \frac{p_{p}\left(K^{a}, L^{b}\right)}{p_{p}\left(K^{a}\right) \cdot p_{p}\left(L^{b}\right)} .
$$

If the $p_{p}\left(K^{a}, L^{b}\right)=0$, the value of the monomial $p_{p}\left(K^{a}, L^{b}\right) \log \frac{p_{p}\left(K^{a}, L^{b}\right)}{p_{p}\left(K^{a}\right) \cdot p_{p}\left(L^{b}\right)}$ is 0 . If one column of the column-pair is fully conserved, the value is also 0 . The measure is referred to as the MI of physicochemical properties (MIP) and the normalized MIP is defined as:

$$
\operatorname{MIP}^{\prime}(K, L)=\frac{M I P(K, L)}{H_{p}(K, L)},
$$

Where $H_{p}(K, L)=-\sum_{a=1}^{10} \sum_{b=1}^{10} p_{p}\left(K^{a}, L^{b}\right) \log p_{p}\left(K^{a}, L^{b}\right)$.

Moreover, amino acid physicochemical properties are also incorporated to the MIB model. The modified marginal fractional frequency of group $a$ in column $K$ is defined as:

$$
p_{b p}\left(K^{a}\right)=\frac{\frac{p_{p}\left(K^{a}\right)}{q_{p}{ }^{a}}}{\sum_{m=1}^{10} \frac{p_{p}\left(K^{m}\right)}{q_{p}^{m}}} .
$$

Here, $q_{p}$ is the background distribution of physicochemical properties which is based on the BLOSUM62 amino acid background distribution.

Similarly, the covariation of the c_pair $(K, L)$ is calculated as:

$$
\operatorname{MIBP}(K, L)=\sum_{a=1}^{10} \sum_{b=1}^{10} p_{p}\left(K^{a}, L^{b}\right) \log \frac{p_{p}\left(K^{a}, L^{b}\right)}{p_{b p}\left(K^{a}\right) \cdot p_{b p}\left(L^{b}\right)} .
$$

If $p_{p}\left(K^{a}, L^{b}\right)=0$, the value of the monomial $p_{p}\left(K^{a}, L^{b}\right) \log \frac{p_{p}\left(K^{a}, L^{b}\right)}{p_{p}\left(K^{a}\right) \cdot p_{p}\left(L^{b}\right)}$ is considered as 0 . The measure is referred to as the MIP with the physicochemical properties background distribution (MIBP) in the paper. And the normalized MIBP is defined as:

$$
\operatorname{MIBP}^{\prime}(K, L)=\frac{\operatorname{MIP}(K, L)}{H_{p}(K, L)} .
$$

The measures MI', MIB', MIP' and MIBP' are used to quantify covariation in the paper.

\section{conn $(\mathbf{k})$ is a more robust indicator than individual covariation score}

It has been proved that individual MI values may be misleading [39]. In order to improve individual scores, 
[23] introduced an indicator $\operatorname{conn}(k)$ to characterize individual residues. In the paper, the top 75 high-scoring pairs are taken into account. And the cut-off of $\operatorname{conn}(k)$ is 5 in MI', MIB' and a comparing method ELSC. While in MIP' and MIBP', since there are ten groups, we take the top 25 high-scoring pairs and the cut-off of $\operatorname{conn}(k)$ is 3. Throughout this paper, we use $\operatorname{conn}(k)$-name to denote the $\operatorname{conn}(k)$ score of the 'name' method. For example, conn $(k)$-MI' denotes the conn $(k)$ score of MI'.

\section{Results and Discussion}

In order to compare our new methods with existing ones, all chosen methods, namely MI', H2r, ELSC, MIB', MIP' and MIBP' are tested on four MSAs. The first one is a toy MSA is shown in Table 1 with 6 sequences and each sequence has 6 residues. Others are commonly used protein families in comparing coevolution methods, which include 1JXA-A, 1B93-A [28] and PF01053 protein family. We use $1 \mathrm{QGN}$ to denote the related protein sequence of PF01053. Results on these MSAs are shown in Tables 2, 3, 4 and 5 and Figures 1, 2, 3, 4, 5 and 6[40]. However, ELSC is not applied to PF01053, for the method suffers an arithmetic overflow when sequence number is too large.

\section{Comparison of different methods on a toy MSA}

In order to illustrate the effects of new biological constraints, we generate a toy MSA, which is shown in Table 1 . In the MSA, column 1 and 2 are fully conserved which are used to illustrate the effect of the amino acid background distribution; column 3, 4 and 5 are used to illustrate the differences between the MIP' and MIBP' methods; and column 6 is randomly generated. We then ran and compared all the methods on the toy MSA, and the results are listed in Table 2. For convenience, a pair of columns $A$ and $B$ is represented by c_pair $(A, B)$ in the study.

In this section, MI' and MIB' are compared to show the effect of incorporating amino acid background distribution. For column-pairs with one column fully conserved, such as c_pair (1,3), c_pair (1,4) and c_pair $(1,5)$, the MI' scores are 0 , while MIB' can distinguish them

Table $1 \mathrm{~A}$ toy MSA used to illustrate the differences between these referred methods

\begin{tabular}{cccccc}
\hline C1 & C2 & C3 & C4 & C5 & C6 \\
\hline D & A & W & A & E & E \\
D & A & W & A & E & F \\
D & A & W & A & E & D \\
D & A & Y & C & M & D \\
D & A & Y & C & M & T \\
D & A & Y & C & $M$ & T \\
\hline
\end{tabular}

In the table, $\mathrm{Ci}$ denotes the ith column. with different scores $0.138,0.237$ and 0.141 , respectively. When the first column is fully conserved, the joint frequency of $c \_$pair $(1, K)$ is equal to the observed frequency of the residue represented by column $K$. On the other hand, the observed distribution of residues in column $K$ is used as the marginal distribution, therefore the MI' score of $c_{-}$pair $(1, K)$ is 0 . However for MIB', the marginal distribution is the observed distribution modified by the amino acid background distribution, thus, the MIB' scores of these column-pairs are distinguishable. For example, the MI' score of $c_{\text {_pair }}(1,6)$ is 0 while it is 0.003 for MIB'. Although the difference between 0 and 0.003 is not significant, it reveals that MI' and MIB' are essentially different. In general, the MI' scores of column-pairs with one fully conserved column are 0 , while the MIB' method can distinguish them unless both columns are conserved. This is because that the marginal distribution used in the MIB' methods has been modified by the amino acid background distribution. In addition, the columns which are paired with the same column and get equal MI' scores, such as the $c_{-}$pair $(3,6)$, c_pair $(4,6)$ and $c \_$pair $(5,6)$, are also distinguishable in MIB'. Although the distributions of column 3, 4 and 5 are the same in number, the type of amino acids are different. The result uncovers that the amino acid background distribution is meaningful in measuring the relationship between columns. For column-pairs with fully covariant columns, the MIB' scores might be different. For example the MIB' scores for $c_{\text {_ppair }}(3,4)$, c_pair $(3,5)$ and $c \_$pair $(4,5)$ are $1.375,1.279$ and 1.378 , respectively.

Different from methods based on amino acid frequencies, the MIP' measure is based on amino acids physicochemical properties. In detail, the MIP' values of $c_{-}$pair (4,5), c_pair (4,6) and c_pair(5,6) are 0.077, 0.037 and 0.053 , respectively, while the MI' scores are 0.296 . Table 2 shows that the covariation of physicochemical properties of $c_{-}$pair $(4,5)$ is stronger than that of $c_{-}$pair $(4,6)$ and c_pair $(5,6)$, while they are equivalent by MI'. Similar results can be found in the comparison of MIP' and MIBP'. Table 2 also shows that the MIP' score is 0 if one column in the pair is fully conserved while this scenario doesn't appear under the MIBP' measure. It worths noting that the score of $c_{-}$pair $(1,2)$ is 0 under the MI', MIB' and MIP' measures since these two columns are fully conserved. However, MIBP' can estimate the covariation of them and gives a score 33.425 to the column-pair. Another interesting observation is that: although column 3 is not fully conserved, the ten overlapping classifier can not distinguish $\mathrm{W}$ and $\mathrm{Y}$. Thus the column with the fully conserved properties leads to a MIP' score of $c \_$pair $(3, K)$ 0 . So do $G$ and A, I and L [30]. In contrast, under the MIBP' measure, these pairs can be distinguished due to the modification of marginal distribution by the background distribution of physicochemical properties. 
Table 2 The MI', MIB', MIP' MIBP' and ELSC scores based on the toy MSA

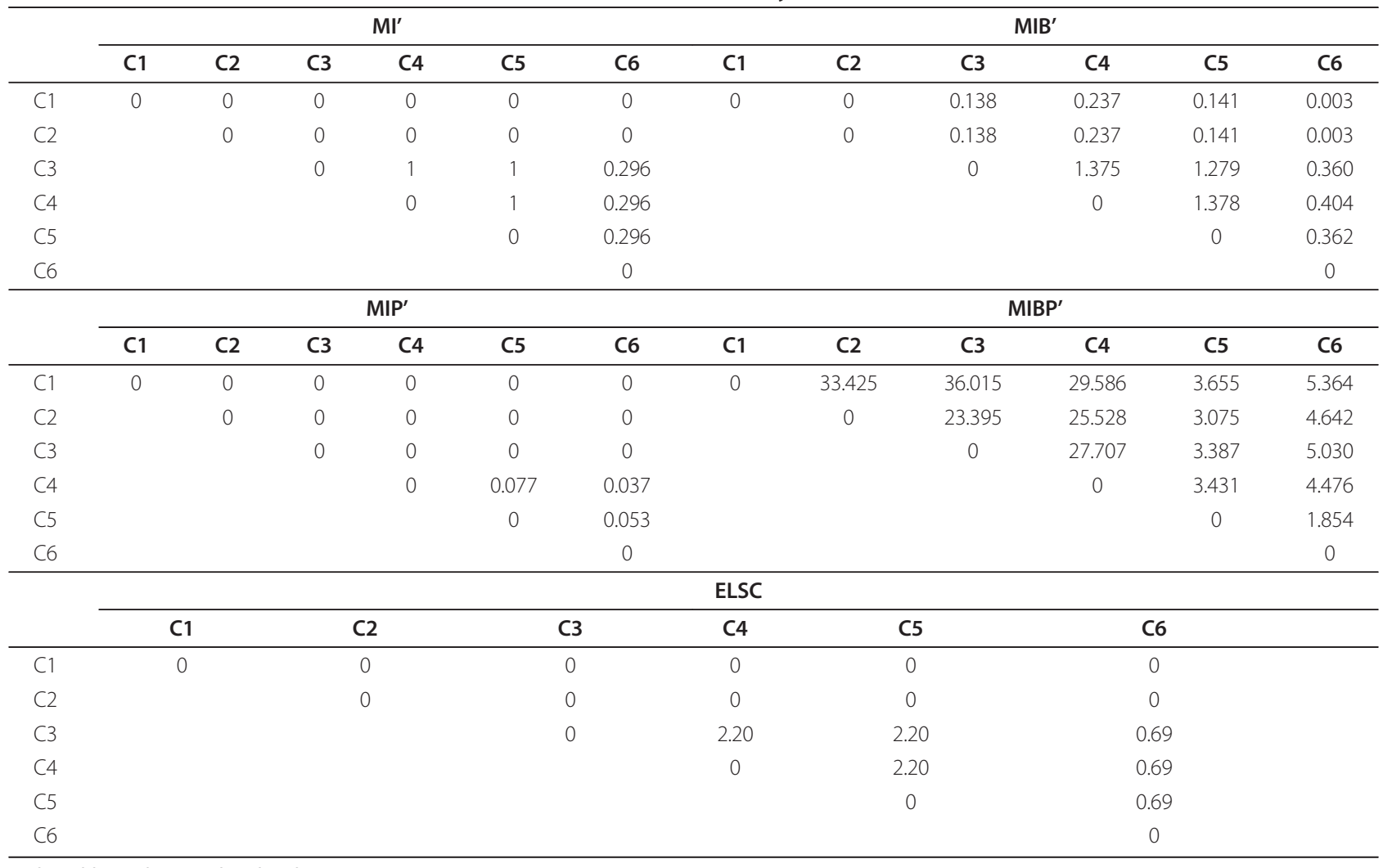

In the table, Ci denotes the ith column.

The effect of incorporating amino acid background distribution

In order to test the effect of incorporating amino acid background distribution, MIB' is compared with MI' and $H 2 r$ on the PF01053 protein family. We plot the relatively high conn $(k)$-MIB' scored sites in Figure 5 and list the detailed information in Table 3. Table 3 shows that the differences among MIB', MI' and $H 2 r$ are obvious. For example, the highest conn( $k$-MIB' site is site 389, while that of MI' and $H 2 r$ is site 388 .

In addition, the number of the sites with $\operatorname{conn}(k)$ MI'> $=5$ is greater than that of MIB' and H2r. Moreover, site 389, a functional important site, gets the highest conn(k)-MIB', but its conn(k)-H2r value is less than 5 . It is already known that in the models of the substrate tCGS (cystathionine $\gamma$-synthase from Nicotiana tabacum) complex (1QGN) [41], the movement of O3' from a mainly hydrophobic environment, arounds site 389 (Phe). Furthermore, sites 239 (Phe) and 211 (Asn), which are essential for maintaining functional environment, are ranked as the first and third sites interlinked with site 389 by the MIB' method. As shown in Figure 2 , sites, which get have relatively high MIB' scores with site 389, form a network around it. It demonstrates that strongly covariation sites surround important functional
Table 3 Comparisons of the $\mathrm{H} 2 \mathrm{r}, \mathrm{MI}^{\prime}, \mathrm{MIB}^{\prime}, \mathrm{MIP}^{\prime}$ and MIBP' methods based on the PF01053 family

\begin{tabular}{|c|c|c|c|c|c|c|c|c|c|}
\hline \multicolumn{2}{|c|}{$H 2 r$} & \multicolumn{2}{|r|}{$\mathrm{Ml}^{\prime}$} & \multicolumn{2}{|c|}{ MIB' } & \multicolumn{2}{|c|}{ MIP' } & \multicolumn{2}{|c|}{ MIBP' $^{\prime}$} \\
\hline$k$ & $\operatorname{conn}(k)$ & $k$ & conn $(k)$ & $k$ & conn $(k)$ & $k$ & $\operatorname{conn}(k)$ & $k$ & $\operatorname{conn}(k)$ \\
\hline 388 & 10 & 388 & 10 & 389 & 30 & 265 & 5 & 236 & 8 \\
\hline 305 & 6 & 272 & 8 & 388 & 9 & 236 & 5 & 261 & 8 \\
\hline 276 & 6 & 313 & 8 & 393 & 8 & 261 & 5 & 313 & 6 \\
\hline 307 & 5 & 207 & 7 & 391 & 8 & 313 & 4 & 272 & 6 \\
\hline 389 & 5 & 138 & 7 & 268 & 7 & 423 & 4 & 423 & 5 \\
\hline 285 & 4 & 393 & 6 & 386 & 7 & 272 & 4 & 265 & 5 \\
\hline 386 & 4 & 236 & 6 & 305 & 7 & 427 & 4 & 207 & 5 \\
\hline \multirow[t]{9}{*}{389} & 4 & 268 & 6 & 280 & 6 & 207 & 4 & 427 & 4 \\
\hline & & 265 & 6 & 402 & 5 & & & & \\
\hline & & 261 & 6 & 308 & 5 & & & & \\
\hline & & 391 & 6 & 211 & 5 & & & & \\
\hline & & 389 & 5 & & & & & & \\
\hline & & 386 & 5 & & & & & & \\
\hline & & 305 & 5 & & & & & & \\
\hline & & 108 & 5 & & & & & & \\
\hline & & 211 & 5 & & & & & & \\
\hline
\end{tabular}

In the table, $k$ denotes the $k$ th site of the 1 QGN chain. 
Table 4 Comparisons of the H2r, ELSC, MI', MIB', MIP' and MIBP' methods based on the 1JXA-A family

\begin{tabular}{|c|c|c|c|c|c|c|c|c|c|c|c|}
\hline \multicolumn{2}{|c|}{$\mathrm{H} 2 \mathrm{r}$} & \multicolumn{2}{|c|}{ ELSC } & \multicolumn{2}{|c|}{$\mathrm{Ml}^{\prime}$} & \multicolumn{2}{|c|}{ MIB' } & \multicolumn{2}{|c|}{ MIP' } & \multicolumn{2}{|c|}{ MIBP' } \\
\hline$k$ & conn $(k)$ & $k$ & $\operatorname{conn}(k)$ & $k$ & conn (k) & $k$ & $\operatorname{conn}(k)$ & $k$ & conn $(k)$ & $k$ & $\operatorname{conn}(k)$ \\
\hline 329 & 9 & 329 & 12 & 32 & 13 & 313 & 12 & 87 & 7 & 504 & 8 \\
\hline 331 & 8 & 102 & 7 & 29 & 12 & 351 & 8 & 78 & 7 & 350 & 8 \\
\hline 102 & 6 & 27 & 6 & 73 & 11 & 503 & 8 & 84 & 7 & 481 & 6 \\
\hline 332 & 5 & 3 & 6 & 84 & 11 & 329 & 7 & 86 & 6 & 485 & 6 \\
\hline 535 & 5 & 2 & 6 & 86 & 11 & 331 & 6 & 73 & 6 & 598 & 5 \\
\hline \multirow[t]{7}{*}{532} & 4 & 32 & 5 & 87 & 11 & 539 & 6 & 99 & 6 & 396 & 5 \\
\hline & & & & 78 & 11 & 502 & 6 & 29 & 4 & 354 & 5 \\
\hline & & & & 27 & 10 & 602 & 5 & 123 & 4 & 403 & 4 \\
\hline & & & & 123 & 10 & 254 & 5 & & & 603 & 3 \\
\hline & & & & 99 & 10 & 332 & 5 & & & & \\
\hline & & & & 125 & 8 & & & & & & \\
\hline & & & & 26 & 7 & & & & & & \\
\hline
\end{tabular}

In the table, $k$ denotes the $k$ th site of the 1JXA-A chain.

domains to make a compensatory effect to maintain the function of the protein, a conjecture being validated by many case studies [5,13,39]. These case studies are also tested on the 1B93-A family and the general results are similar.

Besides $H 2 r$ and MI', MIB' was also compared with the ELSC method proposed by [26]. ELSC uses a perturbation-based algorithm for calculating explicit likelihood of subset covariance, however the background distribution is also ignored. ELSC and MI' are similar since the background distribution is not taken into account for both methods. As shown in Table 4, the sites with high conn(k)-MIB' and those of ELSC are different. Only site 329 is predicted by both methods. Besides, the sites with high $\operatorname{conn}(k)$-MIB' scores are mostly in the $\mathrm{N}$ terminal while that of ELSC are dispersed. On the 1B93-A family the ELSC shows no significant advantage over other methods. Moreover, it suffers arithmetic overflow when sequence number of the MSA is too large. In general, these results suggest that incorporating amino acid background is very important in measuring coevolution.

\section{Coevolution of amino acid physicochemical properties}

In contrast to previous methods which consider amino acids as symbols in a uniformly diverse alphabet, MIP' and MIBP' try to account for amino acid physicochemical properties. As shown in Table 3, the sites predicted by MIP' and MIBP' are different to those by MI' and MIB'. The highest conn(k)-MI' site is site 388, while that of MIP' and MIBP' is site 265 and 236, respectively. In addition, some sites predicted by MIP' and MIBP' are also predicted by MI', but not by MIB'. These results demonstrate that the classification of amino acids and physicochemical properties are different in depicting the MSA. Moreover, site 236 and 261 in PF01053 family, site 504, 481, 485 and 603 in 1JXA-A family, and site 19 and 91 in 1B93-A family are catalytic residues. It means that there are physicochemical properties based networks to maintain the catalytic environment or support the catalytic process.

An in-depth analysis on PF01053 family is also given to show the performances of MIBP'. It has been proven that the carboxylate OD2 of site 236 stabilizes the positively charged pyridine nitrogen of PLP [41]. Site 261 takes its role in catalysis by tCGS named ping-pong mechanism in the first and final steps. They coevolve to maintain specific physicochemical environment stabilization in or around the active region. As shown in Table 4 , the high conn $(k)$-MIBP' scored sites are in the Nterminal of the sequence while that of the MIP' are in the $\mathrm{C}$-terminal. It demonstrates that the results are affected by background distribution significantly. Moreover, four of five catalytic residues are highly scored in the 1JXA-A family and the related structure [42]. Catalytic sites are located in the $\mathrm{N}$-terminal half of the domain at the carboxyl edge of the $\beta$-sheet. And the sites with high conn(k)-MIBP' scores are in the isomerase domain of GlmS (248-608) which is responsible for the binding of Fru6P and its conversion to GlcN6P. However site 504 is separated to other high scoring sites in space. In [43], it was shown that residue 504 plays a key role in the sugar ring opening on a different polypeptide chain. A Schiff base with residue 603 is formed and it is replaced by the incoming ammonia in the Schiff base. It also indicates that the mutation of residue 603 from Lys to Arg results in a decreasing of the synthase activity and an increasing of the isomerase activity. Site 485 forms $\mathrm{H}$ bond with the hydroxyl groups of the sugar.

To compare with the conservation information, the JSD method [28] is used to rank the degree of sites in the 1JXA-A family at different identity thresholds and 
Table 5 Comparisons of the H2r, MI', MIB', MIP' and MIBP' methods based on the 1B93-A family

\begin{tabular}{|c|c|c|c|c|c|c|c|c|c|c|c|}
\hline \multicolumn{2}{|c|}{$H 2 r$} & \multicolumn{2}{|c|}{ ELSC } & \multicolumn{2}{|c|}{$\mathrm{Ml}^{\prime}$} & \multicolumn{2}{|c|}{ MIB' } & \multicolumn{2}{|c|}{ MIP' } & \multicolumn{2}{|c|}{ MIBP' $^{\prime}$} \\
\hline$k$ & conn $(k)$ & $k$ & conn $(k)$ & $k$ & conn $(k)$ & $k$ & conn $(k)$ & $k$ & conn $(k)$ & $k$ & conn $(k)$ \\
\hline 90 & 10 & 103 & 15 & 90 & 13 & 90 & 22 & 42 & 8 & 91 & 8 \\
\hline 29 & 8 & 29 & 10 & 103 & 9 & 29 & 15 & 67 & 6 & 23 & 7 \\
\hline 110 & 7 & 110 & 9 & 129 & 8 & 109 & 9 & 131 & 3 & 19 & 6 \\
\hline 103 & 6 & 90 & 8 & 67 & 8 & 104 & 7 & 16 & 3 & 48 & 6 \\
\hline 130 & 6 & 78 & 8 & 132 & 7 & 67 & 7 & & & 45 & 6 \\
\hline \multirow[t]{8}{*}{109} & 5 & 71 & 8 & 110 & 7 & 93 & 6 & & & 70 & 6 \\
\hline & & 99 & 8 & 71 & 7 & 97 & 6 & & & 69 & 6 \\
\hline & & 109 & 7 & 130 & 7 & 71 & 6 & & & 123 & 3 \\
\hline & & 104 & 6 & 131 & 6 & 103 & 5 & & & & \\
\hline & & 89 & 6 & 29 & 6 & 110 & 5 & & & & \\
\hline & & 67 & 6 & 104 & 6 & 99 & 5 & & & & \\
\hline & & 97 & 5 & 109 & 5 & & & & & & \\
\hline & & 65 & 5 & 65 & 5 & & & & & & \\
\hline
\end{tabular}

In the table, $k$ denotes the $k$ th site of the 1B93-A chain.

the related results are shown in the supplement material (Additional files 1, 2, 3 and 4). As shown in Table 6, the sites with high conn $(k)$ scores are not highly scored by the JSD. Among the sites predicted by MI', only sites 86 and 84 are in the top ten of the JSD rank. And in the MIB', the sites are less conserved than MI'. Moreover, for MIP' and MIBP', although the sites are relatively conserved, the JSD rank can not correspond to the conn (k) value. In general, the relevance of conservation and covariation is not so high, which demonstrates that the conservation and covariation information are relatively independent properties of proteins.

\section{Relations between different measures}

A comparison of the MI', MIB', MIP' and MIBP' measures was given based on the MSA of PF01053 and the

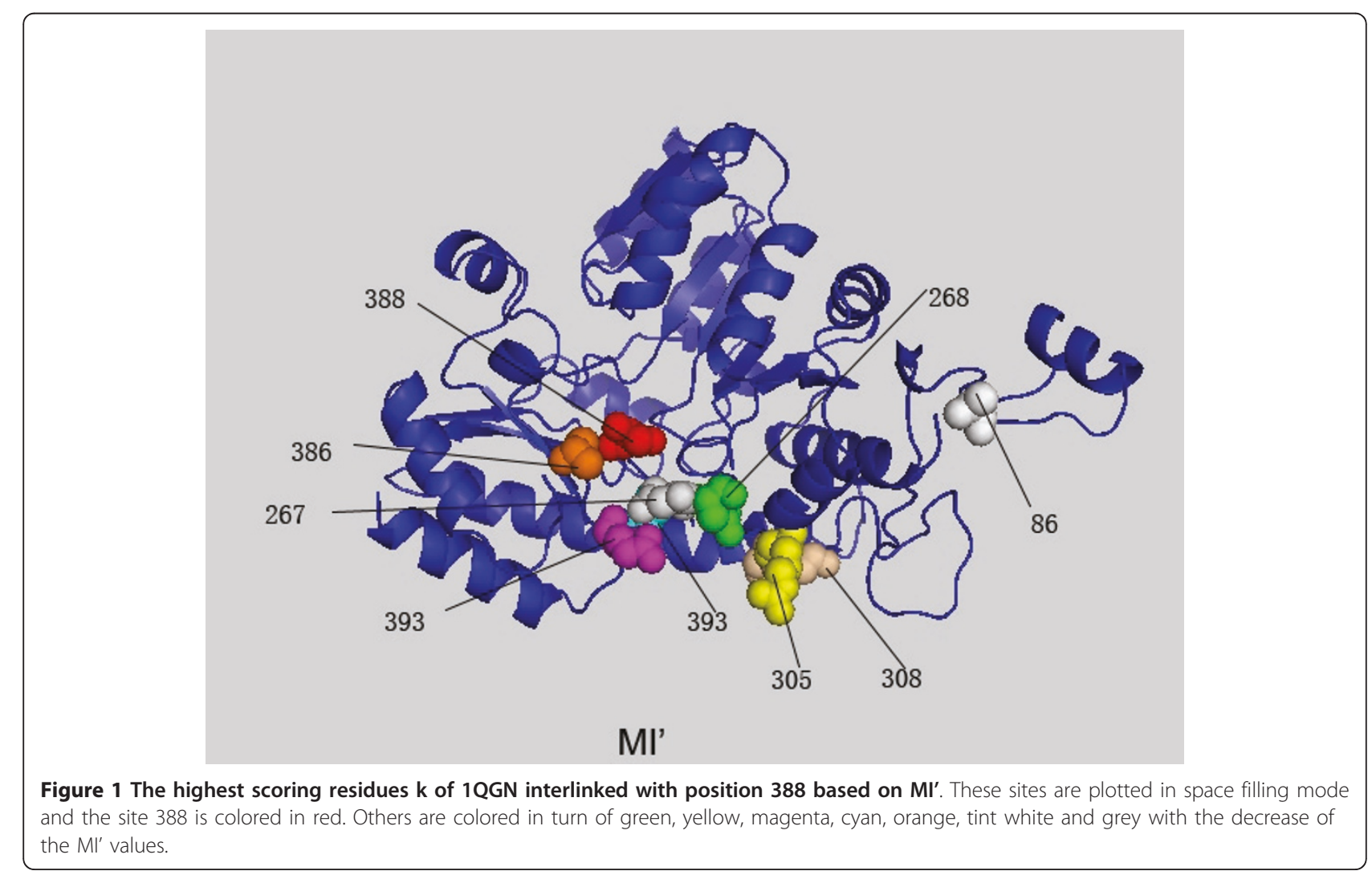




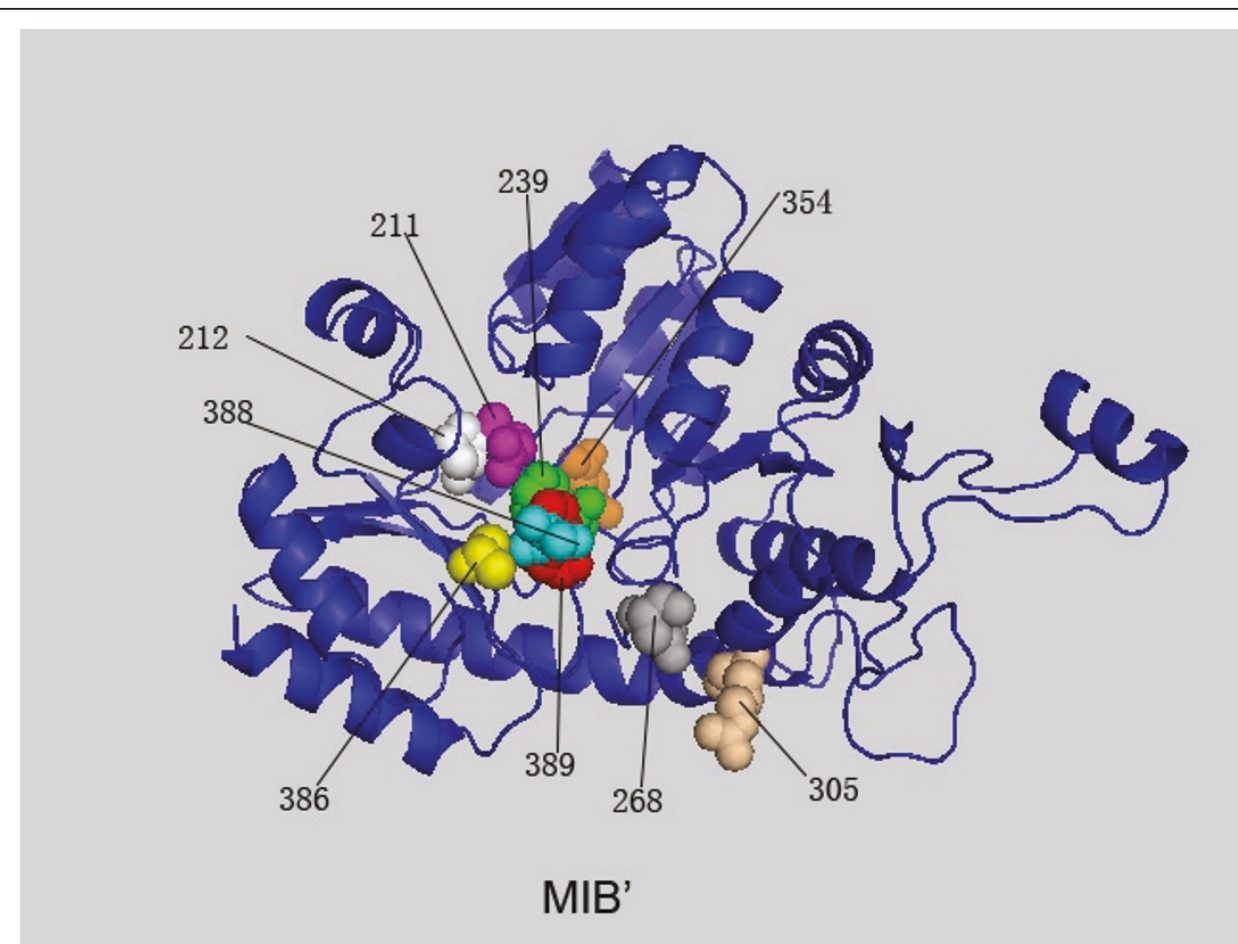

Figure 2 The highest scoring residues $\mathbf{k}$ of 1 QGN interlinked with position $\mathbf{3 8 9}$ based on MIB'. These sites are plotted in space filling mode and the site 389 is colored in red. Others are colored in turn of green, yellow, magenta, cyan, orange, tint white and grey with the decrease of the MIB' values.

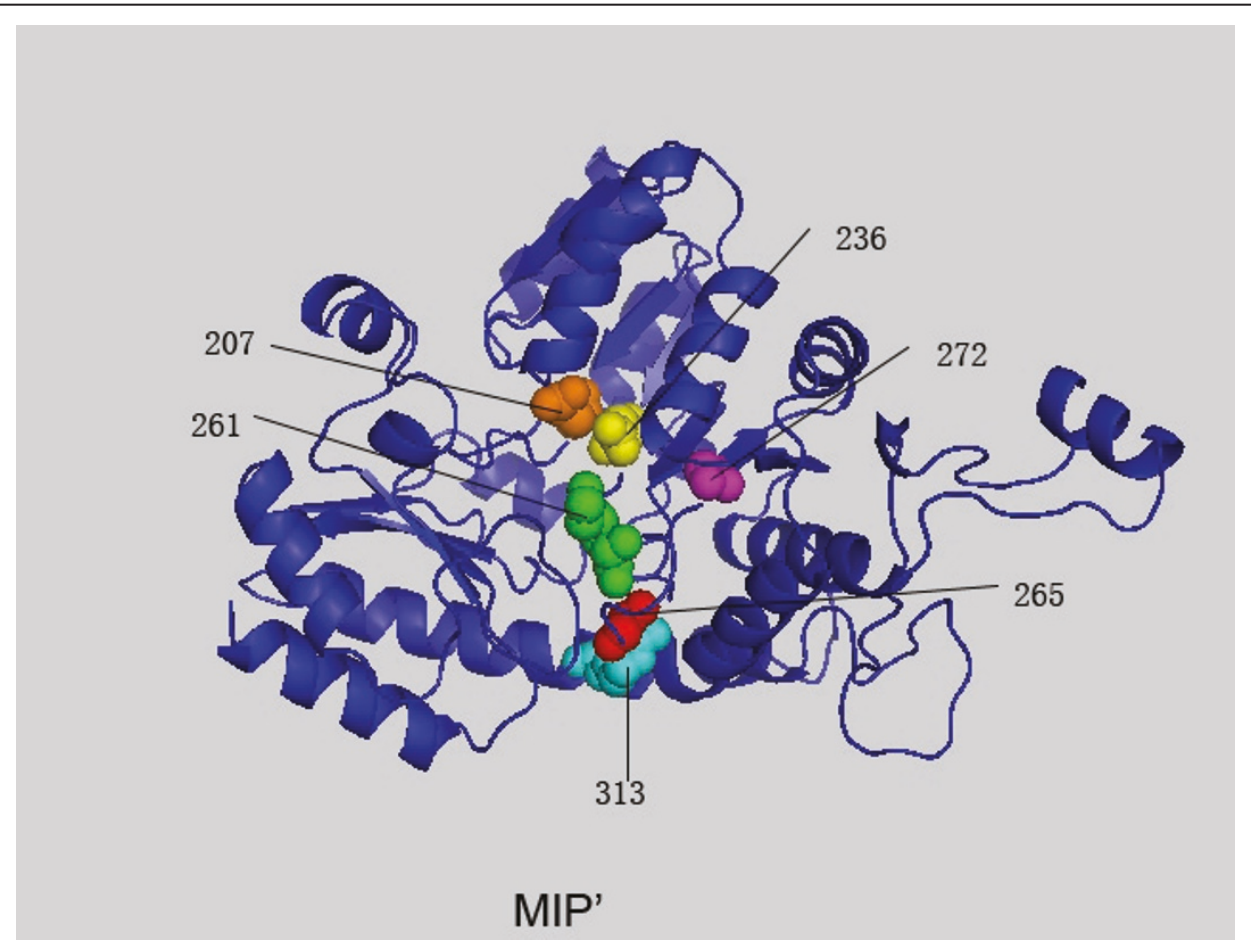

Figure 3 The highest scoring residues $\mathbf{k}$ of 1QGN interlinked with position 265 based on MIP'. These sites are plotted in space filling mode and the site 265 is colored in red. Others are colored in turn of green, yellow, magenta, cyan, orange, tint white and grey with the decrease of the MIP' values. 


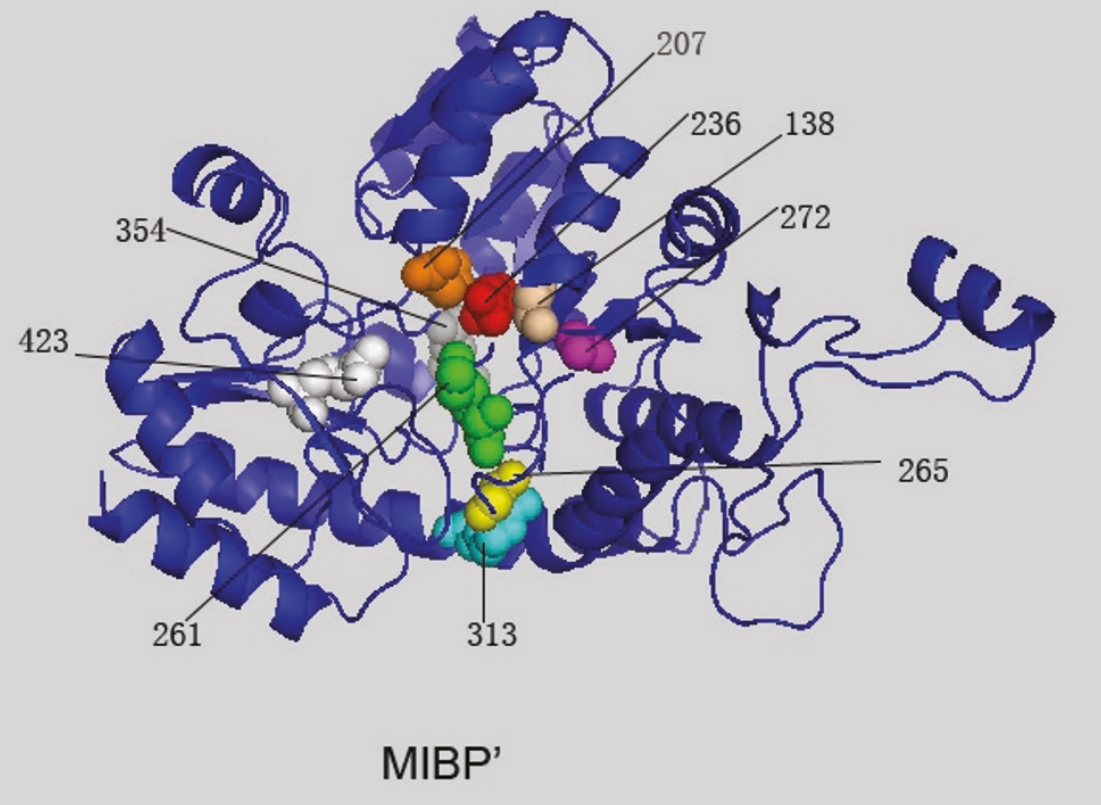

Figure 4 The highest scoring residues $\mathbf{k}$ of $1 \mathrm{QGN}$ interlinked with position $\mathbf{2 3 6}$ based on MIBP'. These sites are plotted in space filling mode and the site 236 is colored in red. Others are colored in turn of green, yellow, magenta, cyan, orange, tint white and grey with the decrease of the MIBP' values.

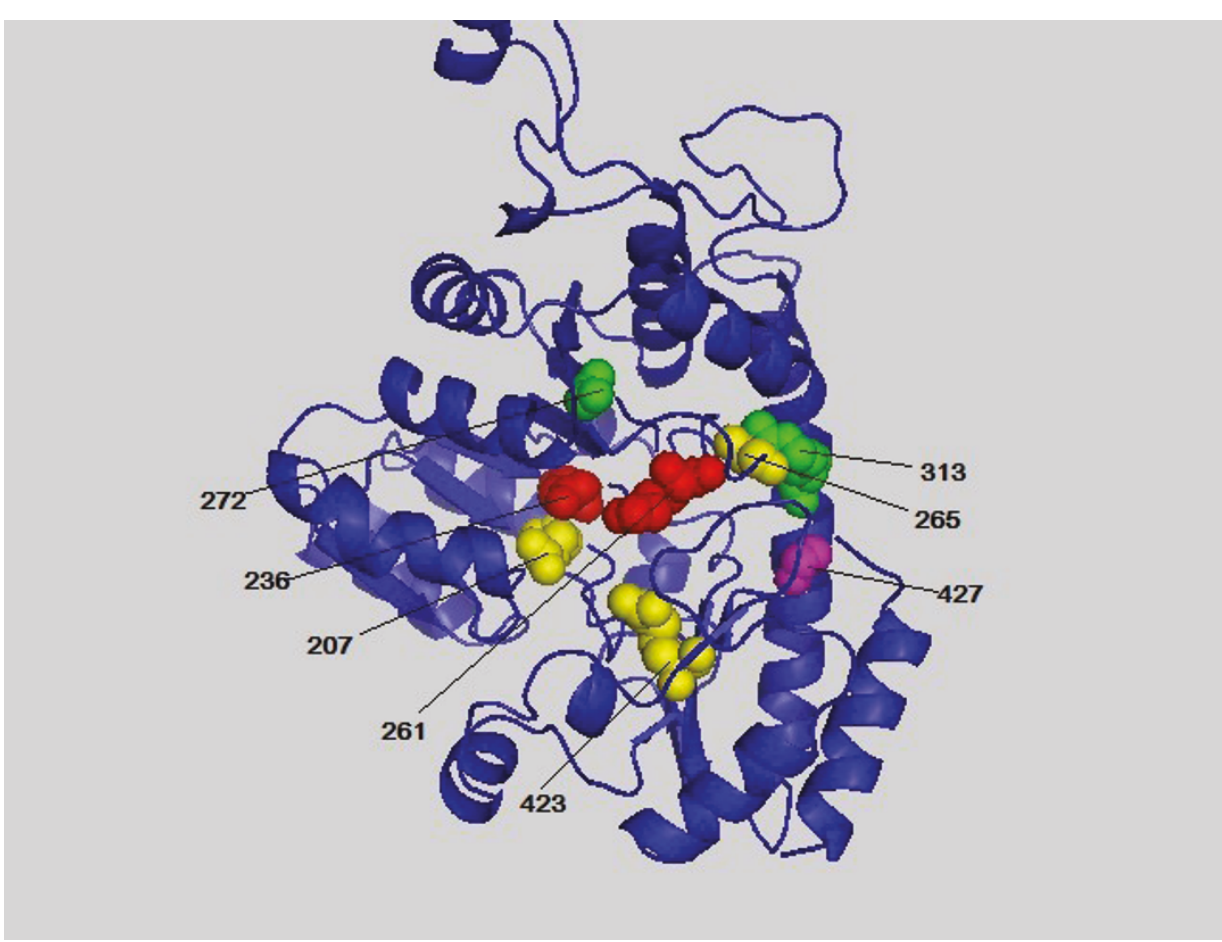

Figure 5 The highest scored residues of $1 Q$ QGN based on the conn(k)-MIBP' scores. Residues of the $1 \mathrm{QGN}$ with conn(k)-MIBP' scores $\geq 4$ are plotted in space filling mode and labeled. The sites 236 and 261 with the highest score 8 are colored in red. In addition, they are catalytic sites of the chain. Other chosen residues are colored in turn of green, yellow and magenta with the decrease of the conn(k)-MIBP' scores. 


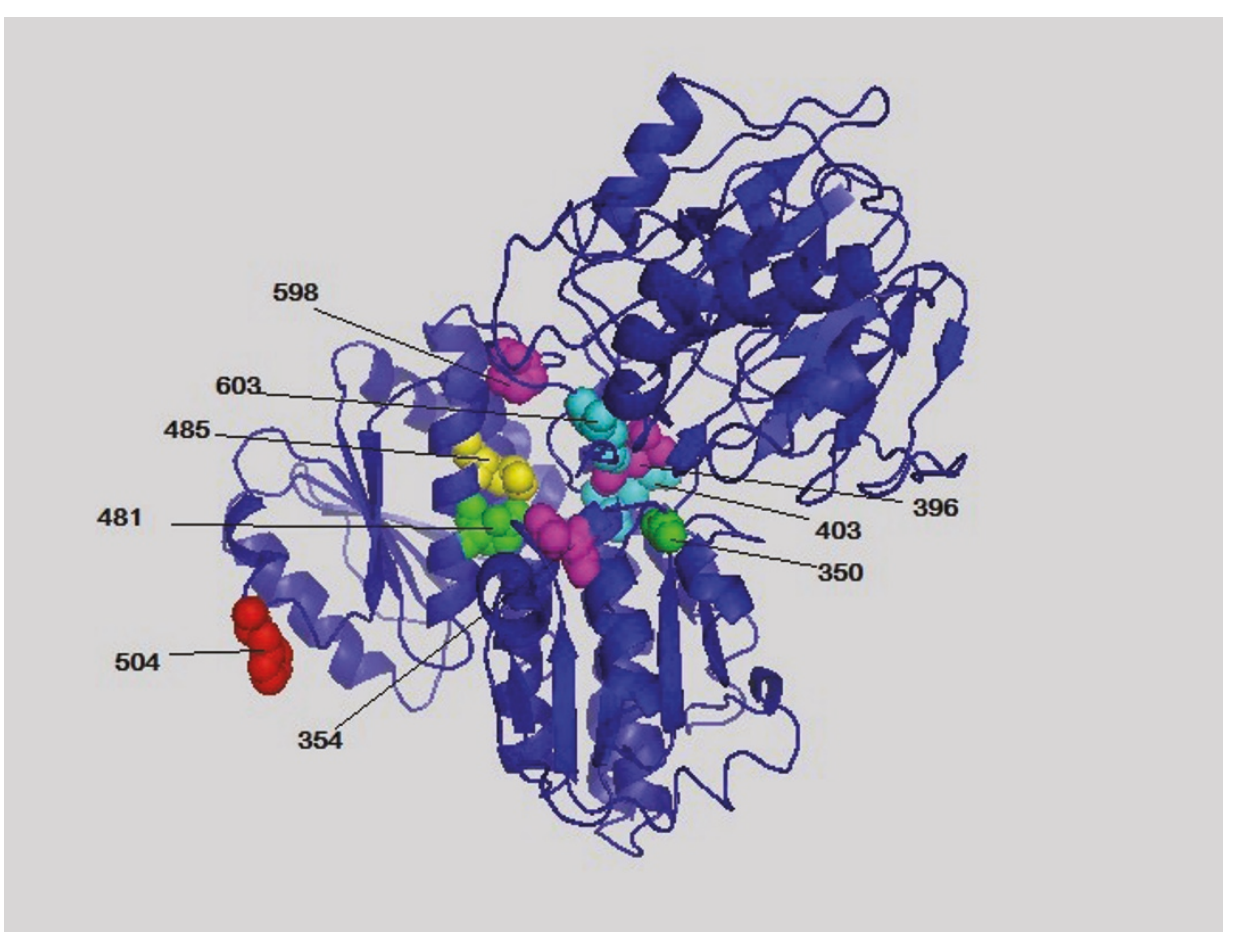

Figure 6 The highest scored residues of 1JXA-A based on the conn(k)-MIBP' scores. Residues of the 1JXA-A with conn(k)-MIBP' scores $\geq 3$ are plotted in space filling mode and labeled. The site 504 with the highest score 8 is colored in red. Other chosen residues are colored in turn of green, yellow, magenta and cyan with the decrease of the conn( $k$ )-MIBP' scores.

related protein structure is PDB ID: 1QGN[28]. In Figures 1, 2, 3 and 4 we found that with different biological constraints, the highest scoring sites are different and the comutating sites are also different. In the MI' method, the highest scoring site is 388 while that of MIB', MIP' and MIBP' are 389, 265 and 236, respectively. On the other hand, to make certain differences between these measures, we used the Friedman test to judge whether the performance statistics for these measures are significantly different. The difference between the performances of two measures is called statistically significant if the $P$-value estimated by the Friedman test with Bonferroni correction is less than 0.05 [28].

To testify the validity of the amino acid background distribution, the MI' and MIB' measures were compared. As shown in Table 7, the P-value between MI' and MIB' is $6.82 \times 10^{-4}$, thus there is significant difference

Table 6 Comparison of conservation method and coevolution methods based on 1JXA-A family

\begin{tabular}{|c|c|c|c|c|c|c|c|}
\hline conn(k)-Ml' rank & JSD rank & conn(k)-MIB' rank & JSD rank & conn(k)-MIP' rank & JSD rank & conn(k)-MIBP' rank & JSD rank \\
\hline 32 & 64 & 313 & 310 & 87 & 12 & 504 & 2 \\
\hline 29 & 23 & 351 & 28 & 78 & 59 & 350 & 52 \\
\hline 73 & 21 & 503 & 42 & 84 & 10 & 481 & 29 \\
\hline 84 & 10 & 329 & 97 & 86 & 3 & 485 & 27 \\
\hline 86 & 3 & 331 & 288 & 73 & 21 & 598 & 11 \\
\hline 87 & 12 & 539 & 24 & 99 & 56 & 396 & 35 \\
\hline 78 & 59 & 502 & 134 & 29 & 23 & 354 & 16 \\
\hline 27 & 65 & 602 & 73 & 123 & 19 & 403 & 26 \\
\hline 123 & 19 & 254 & 45 & & & 603 & 32 \\
\hline 99 & 56 & 332 & 151 & & & & \\
\hline 125 & 36 & & & & & & \\
\hline 26 & 31 & & & & & & \\
\hline
\end{tabular}

The column of conn( $k$-name rank represents the sites with conn( $k$ ) scores in 'name' method. And the sites are presented from the high conn( $k$ ) scores to low ones. The column of JSD rank represents the corresponding JSD rank of the site. 


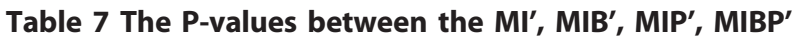
methods

\begin{tabular}{ccccc}
\hline Measure & MI' $^{\prime}$ & MIB' $^{\prime}$ & MIP' $^{\prime}$ & MIBP' $^{\prime}$ \\
\hline MI' $^{\prime}$ & 1 & $6.82 \times 10^{-4}$ & $2.03 \times 10^{-7}$ & $7.7 \times 10^{-2}$ \\
MIB' $^{\prime}$ & & 1 & $9.63 \times 10^{-8}$ & $3.68 \times 10^{-8}$ \\
MIP' $^{\prime}$ & & & 1 & $8.27 \times 10^{-1}$ \\
MIBP' $^{\prime}$ & & & 1 \\
\hline
\end{tabular}

between them. It confirmed the conclusion of [25] that the biological constraints is meaningful in measuring covariation, and evolution pressures of amino acids are removed successfully by using their background distribution. To clarify differences between covariation of amino acids and covariation of amino acid physicochemical properties, the MI' and MIP' measures were also compared. The P-value between the MI' and MIP' measures is $2.03 \times 10^{-7}$. The $P$-value indicates that the covariation of amino acids is significantly different to that of physicochemical properties. These results indicate that the covariation of physicochemical properties is a new aspect of biological information to detect coevolution.

\section{Effect of sequence identity threshold}

The effect of sequence identity thresholds in measuring coevolution is tested in this section. Sequence identity thresholds of $90 \%, 80 \%, 70 \%$ and $60 \%$ are chosen as examples on the 1JXA-A protein family. The identity thresholds $<60 \%$ are ignored since the account of the number of sequences is less than125. Performances of MIB' on the protein family are shown in Table 8 and performances of other methods are shown in the supplement materials. Table 8 shows that sites change significantly with the decreasing of the identity thresholds and the numbers of the predicted sites are also

Table 8 Performances of MIB' at different sequence identity

\begin{tabular}{cccccccc}
\hline \multicolumn{2}{c}{$\mathbf{9 0}$} & \multicolumn{2}{c}{$\mathbf{8 0}$} & \multicolumn{2}{c}{$\mathbf{7 0}$} & \multicolumn{2}{c}{$\mathbf{6 0}$} \\
\hline $\boldsymbol{k}$ & conn(k) & $\boldsymbol{k}$ & conn(k) & $\boldsymbol{k}$ & conn $(\boldsymbol{k})$ & $\boldsymbol{k}$ & conn $(\boldsymbol{k})$ \\
\hline 313 & 12 & 313 & 16 & 313 & 12 & 238 & 24 \\
351 & 8 & 400 & 6 & 238 & 11 & 332 & 8 \\
503 & 8 & 332 & 6 & 502 & 8 & 331 & 7 \\
329 & 7 & 502 & 6 & 331 & 6 & & \\
331 & 6 & 329 & 6 & 332 & 5 & & \\
539 & 6 & & & 329 & 5 & & \\
502 & 6 & & & & & & \\
602 & 5 & & & & & & \\
254 & 5 & & & & & & \\
332 & 5 & & & & & & \\
\hline
\end{tabular}

The MIB' method is tested on the 1JXA-A protein family at sequence identities $90 \%, 80 \%, 70 \%$ and $60 \%$. decreasing. For example, site 313 is the highest conn $(k)$ MIB' scoring site at the identity thresholds of $90 \%, 80 \%$ and $70 \%$, while it is not predicted at the identity threshold of $60 \%$. And at relatively high identity thresholds, site 238 is not predicted while its conn( $k$-MIB' score is high at relatively low identity thresholds. Moreover, there are only 3 sites left at the identity threshold $60 \%$. It means these three sites coevolve with many columns which do not coevolve with other columns strongly. While at $90 \%$ threshold, the information is enough to reveal the covariation of those columns. Thus, it demonstrates that the lower identity threshold affects the covariation significantly. It may lead to the consequence that some sites without coevolved property are mistakenly regard as the highly coevolved sites. In contrast, some sites with coevolved property are not predicted since the signal of coevolution reduces with the lower identity. It is true that sequences with high identity would reduce the correctness of the MSA, thus identity threshold of $95 \%$ or $90 \%$ is acceptable. The related results are submitted with the paper.

\section{Conclusions}

In this study, we propose two new biological constraints, based on which several new measures are designed to detect protein residue coevolution. The first constraint is amino acid background distribution which is used to develop the MIB' method. In contrast to previous methods which focused on how to transform joint count to joint frequency, MIB' method tries to remove the effects of amino acids evolutional pressures in the measure. By incorporating the new biological constraints, we found that MIB' is more effective in measuring amino acid coevolution. The second constraint is the physicochemical properties of amino acids which are used in the MIP' method. Motivated by the MIB' method, MIBP' method which removes the physicochemical properties' evolutional pressures is also proposed. Results show that the MIBP' method is sensible to catalytic sites. It indicates that physicochemical properties of residues around catalytic sites are strongly evolved. Moreover, results show that the MIBP' measure is significantly different from methods based on amino acid distribution. Thus the covariation of physicochemical properties supplies new coevolution information.

\section{Additional material}

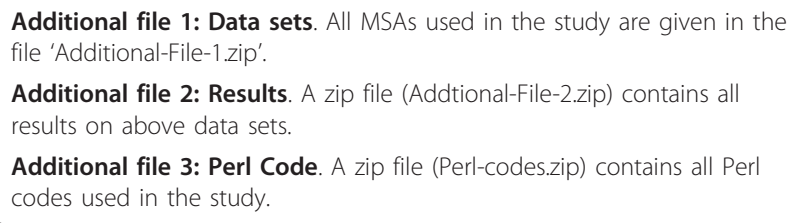


Additional file 4: Effects of sequence identity. The supplement material includes the comparisons of these proposed methods on different sequence identities based on the 1JXA-A family. Their corresponding JSD ranks are also shown in the material.

\section{Acknowledgements}

This work was partially supported by the National Natural Science Foundation of China(No. 10731040), Shanghai Leading Academic Discipline Project(No. S30405) and Innovation Program of Shanghai Municipal Commission(No. 09zz134).

\section{Author details}

${ }^{1}$ School of Mathematical Sciences, Dalian University of Technology, 116024 Dalian, People's Republic of China. ${ }^{2}$ College of Advanced Science and Technology, Dalian University of Technology, 116024 Dalian, People's Republic of China. ${ }^{3} \mathrm{MPI}-\mathrm{CAS}$ Institute of Computational Biology, Chinese Academy of Sciences at Shanghai, 200031 Shanghai, People's Republic of China. ${ }^{4}$ Scientific Computing Key Laboratory of Shanghai Universities, 200234 Shanghai, People's Republic of China. ${ }^{5}$ Department of Mathematics, Shanghai Normal University, 200234 Shanghai, People's Republic of China.

\section{Authors' contributions}

HG carried out the studies on MIB, MIP and MIBP, performed the sequence alignment and drafted the manuscript. YD designed the methods to this study, performed the statistical analysis and helped to draft the manuscript. JY and JW participated in the discussion, and helped to draft the manuscript. All authors have read and approved the final manuscript.

Received: 19 November 2010 Accepted: 26 May 2011

Published: 26 May 2011

\section{References}

1. Wood TC, Pearson WR: Evolution of protein sequences and structures. J Mol Biol 1999, 291(4):977-995.

2. Taylor WR, Hatrick K: Compensating changes in protein multiple sequence alignments. Protein Eng Des Sel 1994, 7(3):341-348.

3. Atwell S, Ultsch M, Vos AMD, Wells JA: Structural Plasticity in a Remodeled Protein-Protein Interface. Science 1997, 278(5340):1125-1128.

4. Chelvanayagam G, Eggenschwiler A, Knecht L, Gonnet G, Benner S: An analysis of simultaneous variation in protein structures. Protein Eng Des Sel 1997, 10:307-316

5. Olivera $L$, Paiva ACM, Vriend G: Correlated mutation analyses on very large sequence families. Chembiochem 2002, 3(10):1010-1017.

6. Martin LC, Gloor GB, Dunn SD, Wahl LM: Using information theory to search for co-evolving residues in proteins. Bioinformatics 2005, 21(22):4116-4124.

7. Goh CS, Bogan AA, Joachimiak M, Walther D, Cohen FE: Co-evolution of proteins with their interaction partners. J Mol Biol 2000, 299(2):283-293.

8. Goh CS, Cohen FE: Coevolutionary Analysis Reveals Insights into Protein Protein Interactions. J Mol Biol 2002, 324(1):177-192.

9. Fares MA: Computational and Statistical Methods to Explore the Various Dimensions of Protein Evolution. CURR BIOINFORM 2006, 1:207-217.

10. Fares MA, McNally D: CAPS: coevolution analysis using protein sequences. Bioinformatics 2006, 22(22):2821-2822.

11. Yip KY, Patel P, Kim PM, Engelman DM, McDermott D, Gerstein M: An integrated system for studying residue coevolution in proteins. Bioinformatics 2008, 24(2):290-292.

12. Pazos F, Helmer-Citterich M, Ausiello G, Valencia A: Correlated mutations contain information about protein-protein interaction. J Mol Biol 1997 271(4):511-523.

13. Weckwerth $W$, Selbig J: Scoring and identifying organism-specific functional patterns and putative phosphorylation sites in protein sequences using mutual information. Biochem Biophys Res Commun 2003, 307:516-521.

14. Choi K, Gomez SM: Comparison of phylogenetic trees through alignment of embedded evolutionary distances. BMC Bioinformatics 2009, 10:423.
15. Pollock DD, Taylor WR, Goldman N: Coevolving protein residues: maximum likelihood identification and relationship to structure. J Mol Biol 1999, 287(1):187-198.

16. Dimmic MW, Hubisz MJ, Bustamante CD, Nielsen R: Detecting coevolving amino acid sites using Bayesian mutational mapping. Bioinformatics 2005, 21(suppl 1):126-135.

17. Fukami-Kobayashi K, Schreiber D, Benner S: Detecting Compensatory Covariation Signals in Protein Evolution Using Reconstructed Ancestral Sequences. J Mol Biol 2002, 319:729-743.

18. Silviu G: Information Theory with Applications McGraw-Hill, New York; 1977.

19. Chakrabarti S, Panchenko A: Coevolution in defining the functional specificity. Proteins 2009, 75(1):231-240

20. Yeang $\mathrm{CH}$, Haussler D: Detecting coevolution in and among protein domains. PLoS Comput Biol 2007, 3:e211.

21. Codoñer FM, Fares MA: Why should we care about molecular coevolution? Evolutionary Bioinformatics Online 2008, 4:29-38.

22. Nimrod G, Glaser F, Steinberg D, Ben-Tal N, Pupko T: In silico identification of functional regions in proteins. Bioinformatics 2005, 21:i328-27.

23. Merkl R, Zwick M: H2r: Identification of evolutionary important residues by means of an entropy based analysis of multiple sequence alignments. BMC Bioinformatics 2008, 9(1):151.

24. Kalinina OV, Novichkov PS, Mironov AA, Gelfand MS, Rakhmaninova AB: SDPpred: a tool for prediction of amino acid residues that determine differences in functional specificity of homologous proteins. Nucleic Acids Res 2004, 32:424-428.

25. Fernandes $A D$, Gloor $G B$ : Mutual information is critically dependent on prior assumptions: would the correct estimate of mutual information please identify itself? Bioinformatics 2010, 26(6):1135-1139.

26. Dekker JP, Fodor A, Aldrich RW, Yellen G: A perturbation-based method for calculating explicit likelihood of evolutionary covariance in multiple sequence alignments. Bioinformatics 2004, 20(10):1565-1572

27. Dou $Y$, Zheng $X$, Wang J: Several appropriate background distributions for entropy-based protein sequence conservation measures. I theor Biol 2009, 262(2):317-322.

28. Capra JA, Singh M: Predicting functionally important residues from sequence conservation. Bioinformatics 2007, 23(15):1875-1882.

29. Henikoff S, Henikoff JG: Amino Acid Substitution Matrices from Protein Blocks. PNAS 1992, 89:10915-10919.

30. Dou Y, Zheng X, Wang J: Prediction of Catalytic Residues Using the variation of stereochemical properties. PROTEIN J 2009, 28:29-33.

31. Friedberg I, Margalit $\mathrm{H}$ : Persistently conserved positions in structurally similar sequences dissimilar proteins: roles in preserving protein fold and function. Protein Sci 2002, 11(2):350-360.

32. Lockless SW, Ranganathan R: Evolutionarily conserved pathways of energetic connectivity in protein families. Science 1999, 286(5438):295-299.

33. Mirny LA, Shakhnovich El: Universally conserved positions in protein folds: reading evolutionary signals about stability, folding kinetics and function. J Mol Biol 1999, 291(1):177-196.

34. Oliveira L, Paiva PB, Paiva ACM, Vriend G: Identification of functionally conserved residues with the use of entropy-variability plots. Protein 2003, 52(4):544-552

35. Chakrabarti S, Bryant SH, Panchenko A: Functional specificity lies within the properties and evolutionary changes of amino acid. $J$ Mol Biol 2007 373:801-810.

36. Petrova NV, Wu CH: Prediction of catalytic residues using support vector machines with selected protein sequence and structural properties. BMC Bioinformatics 2006, 7:312.

37. Taylor WR: The classification of Amino Acid Conservation. J theor Biol $1986,119(2) \cdot 205-218$

38. Finn RD, Mistry J, Schuster-Böckler B, Griffiths-Jones S, Hollich V, Lassmann T, Moxon S, Marshall M, Khanna A, Durbin R, Eddy SR, Sonnhammer ELL, Bateman A: Pfam: clans, web tools and services. Nucleic Acids Res 2006, 34:D247-D251.

39. Gloor GB, Martin LC, Wahl LM, Dunn SD: Mutual information in protein multiple sequence alignments reveals two classes of coevolving positions. Biochemistry 2005, 44(19):7156-7165.

40. Delano WL: molecular graphics system on World Wide Web. DeLano Scientific, site hosted by Sourceforge.net 2002.

41. Steegborn C, Messerschmidt A, Laber B, Streber W, Huber R, Clausen T: The crystal structure of cystathionine $\gamma$-synthase from nicotiana tabacum 
reveals its substrate and reaction specificity. J Mol Biol 1999, 290(5):983-996.

42. Teplyakov A, Obmolova G, Badet B, Badet-Denisot MA: Channeling of ammonia in Glucosamine-6-phosphate synthase. J Mol Biol 2001, 313(5):1092-1102.

43. Laskowski RA, Chistyakov W, Thornton JM: PDBsum more: new summaries and analyses of the known 3D structures of proteins and nucleic acids. Nucleic Acids Res 2005, 33:D266-D268.

doi:10.1186/1471-2105-12-206

Cite this article as: Gao et al:: New methods to measure residues coevolution in proteins. BMC Bioinformatics 2011 12:206.

Submit your next manuscript to BioMed Central and take full advantage of:

- Convenient online submission

- Thorough peer review

- No space constraints or color figure charges

- Immediate publication on acceptance

- Inclusion in PubMed, CAS, Scopus and Google Scholar

- Research which is freely available for redistribution

Submit your manuscript at www.biomedcentral.com/submit
() Biomed Central 